\title{
Azimuthal decomposition of the radiated noise from supersonic shock-containing jets
}

\author{
Marcus H. Wong, ${ }^{1}$, a Rhiannon Kirby, ${ }^{1}$ Peter Jordan, ${ }^{2}$ and Daniel Edgington-Mitchell ${ }^{1}$ \\ ${ }^{1}$ Laboratory for Turbulence Research in Aerospace and Combustion (LTRAC), Department of Me- \\ chanical and Aerospace Engineering, Monash University, Melbourne, VIC 3800, Australia \\ ${ }^{2}$ Département Fluides, Thermique, Combustion, Institut PPrime, CNRS - Université de Poitiers - \\ ENSMA, 86000 Poitiers, France
}

Acoustic measurements of unheated supersonic underexpanded jets with ideally-expanded Mach numbers of 1.14, 1.38 and 1.50 are presented. Of the three components of supersonic jet noise, the focus is on the broadband shock-associated noise (BBSAN) component. Motivated by the modelling of BBSAN using the wavepacket framework, a traversable microphone ring is used to decompose the acoustic pressure into azimuthal Fourier modes. Unlike noise radiated downstream, BBSAN is dominated by azimuthal modes 1 through 3 which are approximately $3-4 \mathrm{~dB} /$ St stronger than the axisymmetric component. Crucially, the relative contribution of successive modes to BBSAN is sensitive to observer angle and jet operating condition. Four azimuthal modes are necessary to reconstruct the total BBSAN signal to within $1 \mathrm{~dB} / \mathrm{St}$ accuracy for the conditions presented here. The analysis suggests, however, that the number of modes required to maintain this accuracy increases as the peak frequency shifts upward. The results demonstrate the need to carefully consider the azimuthal content of BBSAN when comparing acoustic measurements to predictions made by jet noise models built on instability theory.

(C)2020 Acoustical Society of America. [https://doi.org(DOI number)]

$[\mathrm{XYZ}]$

Pages: $1-12$

\section{INTRODUCTION}

Broadband shock-associated noise (BBSAN) is a high-frequency noise component emanating from shockcontaining supersonic jets. Observed in both military (Vaughn et al., 2018) and civilian aircraft (Huber et al., 2014), it is more prominent in the upstream and sideline directions. BBSAN is produced by the interaction of downstream-travelling turbulent structures with the train of shock-cells in the jet plume (Tam, 1995). Unlike the discrete component known as screech (EdgingtonMitchell, 2019; Raman, 1999), where noise is generated by an easily-disrupted feedback loop, suppression of BB$\mathrm{SAN}$ is not straightforward. The lack of simple noise mitigation strategies means BBSAN remains an important component of jet noise.

The work by Harper-Bourne and Fisher (1973) identified the main features of BBSAN; the sound field is characterised by a broad spectral lobe which varies in frequency as a function of observer position and the ideallyexpanded Mach number $\left(M_{j}\right)$ of the jet. The distinctive directivity arises from the constructive and destructive interference within the axially-extended acoustic source. Using the interpretation of Tam and Tanna (1982), the shock-cell structure can be represented as a series of waveguide modes with wavenumbers $k_{n}$ and shock-cell length approximated by $L_{1}=2 \pi / k_{1}$. Tam and Tanna

amarcus.wong@monash.edu
(1982) also showed that the primary BBSAN peak frequency, $f_{p}$, varies with observer angle $\theta$, measured from the downstream jet axis, according to

$$
f_{p}=\frac{u_{c}}{L_{n}\left(1-M_{c} \cos (\theta)\right)}, n=1
$$

where $u_{c}$ is the convection velocity of the large-scale coherent structures, and $L_{1}$ is the shock-cell spacing of the first shock-cell mode $n=1$. Eq. 1 can also be used to predict peaks generated by higher-order shock-cell modes $(n \geq 2)$. The width of the BBSAN peak narrows as observer angle increases. The amplitude of BBSAN scales to the fourth power of the off-design parameter, defined as $\beta=\left(M_{j}^{2}-M_{d}^{2}\right)^{1 / 2}$ where $M_{d}$ is the design Mach number of the nozzle.

When studying noise generated from a circular nozzle, it is useful to exploit the symmetry inherent in the flow. By casting the problem in cylindrical coordinates, both the sound field and its underlying source (Lighthill, 1952) can be decomposed in the azimuthal direction and represented as a Fourier series due to circumferential homogeneity. The advantage of using such a decomposition is exemplified in subsonic jets; the sound field is dominated by the lowest-order azimuthal modes at low polar angles (Cavalieri et al., 2012; Michalke and Fuchs, 1975). The acoustic efficiency of higher-order azimuthal modes is reduced due to azimuthal source interference. The interference is explicitly expressed by the Bessel function term in line-source models (Michalke, 1970). Cavalieri et al. (2012) also showed there exists a direct correspon- 
dence between the acoustic source and sound field of the same azimuthal mode. From a reduced-order modelling perspective, this is appealing, as the desired azimuthal component of the far-field sound can be obtained by computing only the corresponding sound-producing wavepacket (Cavalieri et al., 2019; Jordan and Colonius, 2013).

While our understanding of the role of wavepackets in subsonic and ideally-expanded jets (Sinha et al., 2014) is now relatively mature, their application to model BBSAN is less common. By artificially separating the shock and turbulence components, Tam and Tanna (1982) first proposed the use of spatially-extended instability waves to model the downstream-travelling coherent structures. A BBSAN model (Tam, 1987) was developed based on the interaction of these proposed instability waves with stationary shock-cells; experimental sound spectra were reproduced with a high-degree of accuracy over a wide range of operating conditions. The model predictions, however, were obtained semi-empirically by adopting approximations and scaling arguments. One such simplification was the removal of the dependence on azimuthal wavenumber, treating the source as effectively axisymmetric. Hence, comparisons were only made with the total sound field (Norum and Seiner, 1982). In their model, Ray and Lele (2007) also made a comparable simplification, whereby far-field pressure predictions for only the first three azimuthal modes were combined and scaled to match the total acoustic signal. Recently, wavepacket models for BBSAN have been proposed (Lele, 2005; Wong et al., 2019a,b). Similar to Tam (1987), they have shown fair agreement with experimental spectral shape, but relied on empirical adjustments to match measured amplitudes since no azimuthally-decomposed far-field data was available. The validation of BBSAN models, in particular those predicated on instability theory, is hindered by the lack of azimuthally-decomposed sound-field data.

While azimuthal decomposition has previously been performed on shock-containing jets, the focus has, until now, largely been on the identification of different screech oscillation modes (Edgington-Mitchell, 2019, see references therein). Only a handful of studies have examined the azimuthal modes of BBSAN in detail. Arroyo and Moreau (2019) investigated azimuthal modes using an LES of a non-screeching underexpanded jet at one exit condition. Successive azimuthal modes were found to peak at higher frequencies. Suzuki (2016) also arrived at similar conclusions by observing the azimuthal modal distributions in an LES of an underexpanded jet. An upward shift in emission angle and frequency led to higher-order modes featuring more prominently. Experimentally, this behaviour was also measured by Vold et al. (2012) for an overexpanded jet, although the dependence on observer position was not investigated. Of the above studies, only Suzuki (2016) made mode-bymode comparisons between the decomposed far-field data and BBSAN model predictions, but poor agreement was found. Furthermore, the mentioned studies considered only weakly off-design conditions $(\beta<0.8)$; whether the same observations apply to stronger levels of under- or over-expansion is not known.

The aim of this study is to provide azimuthallydecomposed sound-field measurements of shockcontaining jets for comparison with models. Specifically, we examine the azimuthal modal composition for BBSAN and its variation with Mach number and observer position. The findings should better inform future BBSAN models and allow a basis for comparison between model and experiment. The paper is organised as follows. The experimental facility and setup is discussed in section II, which includes the microphone apparatus, acquisition details and post-processing techniques. In section III, we present both the far-field acoustic results and azimuthally-decomposed mid-field spectra. A summary of the findings and future perspectives is provided in section IV.

\section{METHODOLOGY}

\section{A. Facility Overview}

The acoustic measurements were acquired in the Monash University LTRAC Supersonic Jet Anechoic Facility (SJAF). The facility is designed for the study of acoustics and flow-fields of cold small-scale supersonic jets in a controlled environment. The key components of the facility are presented below.

\section{Anechoic Chamber}

The jet exhausts into an anechoic chamber designed according to the best practices suggested by Ahuja (2003). The interior chamber dimensions (wedge-tip to wedge-tip) are $1.5 \mathrm{~m} \times 1.2 \mathrm{~m} \times 1.4 \mathrm{~m}$. The anechoic chamber walls are acoustically treated using foam wedges with a depth of $400 \mathrm{~mm}$, corresponding to a nominal cut-on frequency of $500 \mathrm{~Hz}$. The floor of the anechoic chamber is also lined with wedge panels. Entrained and exhausted air is replaced via two open sections along the upstream wall. The rectangular openings are acoustically treated with an over-hanging wall to ensure no external noise propagates into the chamber. The acoustic performance of the chamber is provided in Appendix A.

\section{Air Supply}

Compressed air is passed through a heatlessdesiccant dryer which is connected to a $5 \mathrm{~m}^{3}$ air reservoir. The air supply allows for a continuous operation up to a nozzle pressure ratio (NPR) of five. The jet operating condition is determined by the NPR, defined as $N P R=P_{t} / P_{\infty}$, where $P_{t}$ and $P_{\infty}$ are the total and ambient pressures respectively. Since the facility shares the same air supply as a supersonic wind tunnel (Knast and Edgington-Mitchell, 2017), a manual butterfly valve is used to direct air into a large mixing chamber. The air flow is controlled by an eccentric rotary plug control valve. In order to manually select the desired NPR, 
a Fairchild 100 High-Flow Pressure Regulator is used (Edgington-Mitchell et al., 2014). Pressurised air from the mixing chamber is then fed into the jet plenum chamber via a flexible wire-braided high pressure PTFE hose. The stagnation pressure in the plenum, as used to determine NPR, was measured using an RS-3100 pressure transducer, with a range of 0-10 bar. The stated manufacturer accuracy of the transducer is $\pm 0.25 \%$, but the measured range in the plenum was approximately $\pm 1 \%$. The size of the plenum chamber was designed to minimise fluid velocity and to provide a large contraction ratio at the nozzle exit plane. No flow conditioning was used upstream of the nozzle exit. A schematic of the facility is shown in Fig. 1(a).

\section{Nozzle Geometry and Design}

The jet issues from a converging round nozzle which has an exit diameter of $D=8 \mathrm{~mm}$ and a lip thickness of $0.5 \mathrm{~mm}$. The mild-steel plenum chambers and nozzles are assembled in a modular fashion via flange connections (Fig. 1(a)). The modular nature of the jet rig allows for future modifications and upgrades. The jet rig is mounted onto an adjustable steel stand which traverses linear rails to allow constrained axial motion.

The plenum is connected to the nozzle via a bent and straight circular section with a total length of $800 \mathrm{~mm}$ and a diameter of $25.4 \mathrm{~mm}$. The nozzle contour follows a fifth-order polynomial (Morel, 1975) ensuring that a smooth connection with the straight section is achieved. The resulting contraction ratio is 10 .

The small nozzle exit diameter is dictated by the restrictions on air supply and the internal dimensions of the anechoic chamber. Using a small nozzle ensures that the jet is able to operate continuously, even at high NPR. This allows for jet conditions to stabilise, and for lengthy data acquisition. Importantly, the acoustic frequency range of interest is significantly higher than the cut-on frequency of the anechoic chamber.

\section{B. Coordinate Systems}

To facilitate the following discussion, two coordinate systems are used in a similar fashion to Cavalieri et al. (2012) as shown in Fig. 1(b). The cylindrical coordinate system of the jet is represented by $(x, r, \phi)$, where $x$ is the downstream jet axis, $r$ is the radial direction and $\phi$ is in azimuth. This is overlaid with a spherical coordinate system $(R, \theta, \Phi)$ representing the acoustic field, where $R$ is the distance, $\theta$ is the polar angle and $\Phi$ the azimuth.

\section{Operating Conditions}

Acoustic measurements were performed on cold supersonic underexpanded jets. The jet operating conditions are characterised by the NPR and the off-design parameter $\beta$ (Norum and Seiner, 1982). For a converging nozzle, the design Mach number $M_{d}$ is unity, and so $\beta=\left(M_{j}^{2}-1\right)^{1 / 2}$. The Reynolds number at the nozzle exit is of order $10^{5}$ based on the assumption of isentropic flow. We note that the Reynolds numbers of all three conditions correspond to the turbulent transition region of a supersonic jet $\left(R e \approx 10^{5}\right)$. Hence, the measured acoustic field may differ from jets at higher Reynolds numbers which have a fully-turbulent shear layer at the nozzle exit (Bogey et al., 2012; Ozawa et al., 2020). We define $D_{j}$ and $U_{j}$ as the ideally-expanded diameter (Tam and Tanna, 1982) and jet velocity respectively. The operating conditions for the mid-field measurements are summarised in Table I.

TABLE I. Summary of jet operating conditions.

\begin{tabular}{ccccc}
\hline \hline NPR & $M_{j}$ & $D_{j} / D$ & $\beta$ & $R e$ \\
\hline 2.25 & 1.14 & 1.01 & 0.55 & $2.8 \times 10^{5}$ \\
3.10 & 1.38 & 1.05 & 0.95 & $3.8 \times 10^{5}$ \\
3.67 & 1.50 & 1.09 & 1.12 & $4.5 \times 10^{5}$ \\
\hline \hline
\end{tabular}

\section{Data acquisition}

Acoustic measurements were made using G.R.A.S. Type 46BE 1/4" pre-amplified microphones with a frequency range of $4 \mathrm{~Hz}-100 \mathrm{kHz}$ and a nominal sensitivity of $3.6 \mathrm{mV} / \mathrm{Pa}$. The microphone signals were passed through an amplifier and acquired using a National Instruments DAQ. Each recording consisted of 400,000 samples and was acquired at a rate of $200 \mathrm{kHz}$, corresponding to a Nyquist frequency of $100 \mathrm{kHz}$. A low-pass filter was used in the post-processing stage to remove aliasing effects. The microphones were calibrated using a G.R.A.S. pistonphone with a standard calibration level of $114 \mathrm{~dB}$ at $1 \mathrm{kHz}$. The reference pressure used for all acoustic measurements is $P_{r e f}=20 \mu \mathrm{Pa}$. The methodology to obtain acoustic spectra from measured time signals is presented in Appendix B. To facilitate comparison with acoustic measurements of shock-containing jets in literature, nondimensionalised frequency $S t$ is used. We define the Strouhal number as $S t=f D_{j} / U_{j}$ where $f$ is frequency in $\mathrm{Hz}$. For all acoustic measurements, solid surfaces including the ring are covered with acoustic foam (Fig. 1(c)).

\section{E. Microphone Array \& Azimuthal Decomposition}

We obtain sound pressure level readings at two different distances. At a distance of $R=50 D$ from the nozzle exit, which we refer to as 'far-field', noise measurements were obtained at a range of polar angles from $\theta=60^{\circ}-120^{\circ}$. The aim of performing these simple single-microphone far-field measurements is to validate the new facility against trends in the existing literature.

We then present acoustic measurements using an azimuthal ring at a constant radial distance of $r=11 D$ from the jet axis. These measurements are performed to interrogate the modal composition of BBSAN. The 


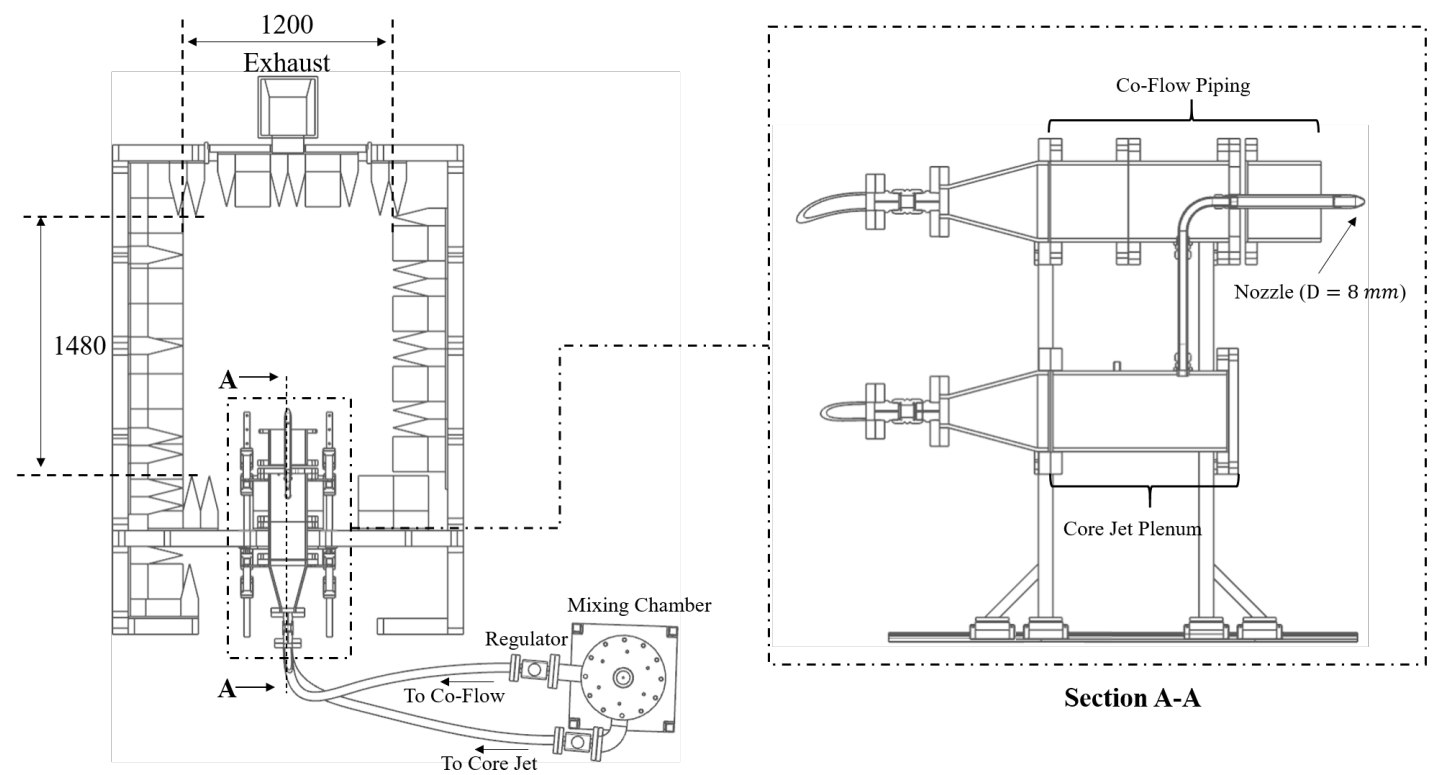

(a)

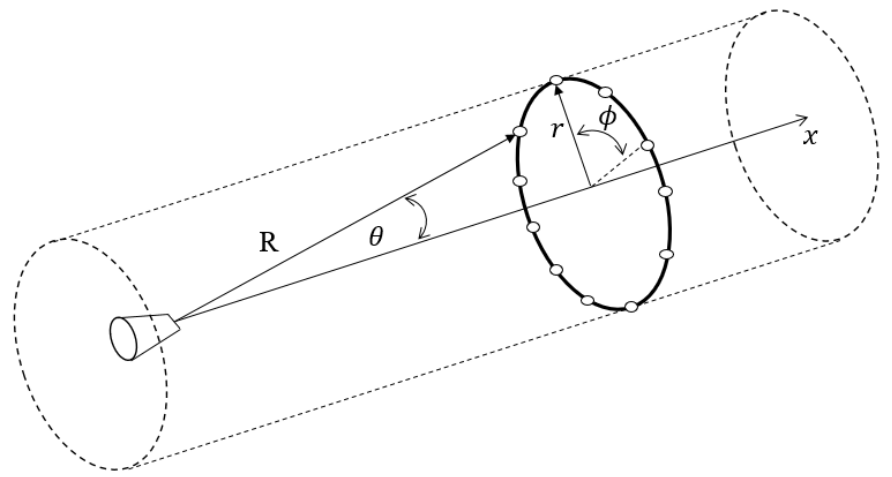

(b)

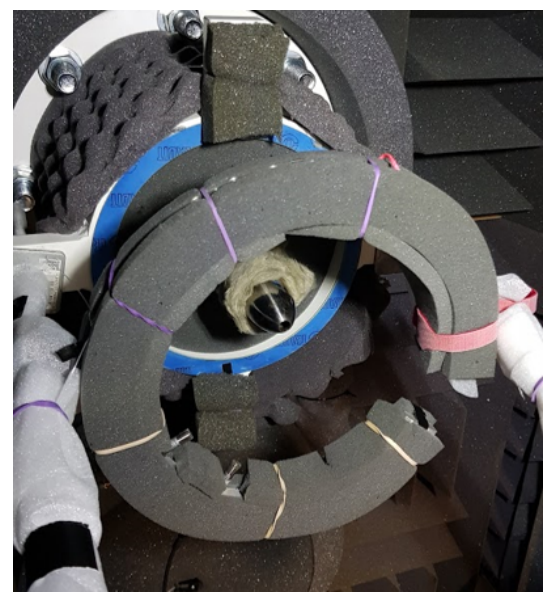

(c)

FIG. 1. Schematics of facility and experimental setup; (a) sectioned plan and side view schematic of SJAF showing key components of the facility (dimensions in $\mathrm{mm}$ ), (b) schematic of adopted coordinate system, (c) azimuthal ring set-up (colour Online) .

azimuthal ring set-up is shown in Fig. 1(c). The location of the microphone is assumed to be well outside the hydrodynamic field for a shock-containing supersonic jet (Seiner and Yu, 1984; Suzuki, 2016; Tam, 1987), ensuring that only acoustic fluctuations are detected. These measurements, which we refer to as 'mid-field', are used to decompose the acoustic field into azimuthal modes using a methodology similar to Sinha et al. (2018).

The methodology involves two microphones, one fixed and the other moved in $10^{\circ}$ azimuthal increments. By tracing the cross-correlation between the two microphones as a function of azimuthal separation, the crosscorrelation matrix $R_{p p}$ is constructed and used to ob- tain the azimuthal decomposition of the radiated acoustic field. The processing steps are detailed in Appendix C. While the process is more time-consuming than for a fixed microphone array (Suzuki and Colonius, 2006), we effectively reproduce the same results with a much smaller microphone count. It was found that sweeping over an azimuthal range of $180^{\circ}$ was sufficient for the frequency range of interest. The azimuthal array is traversed in the axial direction in order to obtain measurements at different polar angles. Since the azimuthal ring is at a fixed distance to the jet axis, the radial distance $R$ between the nozzle and microphone differs for each polar angle. 


\section{EXPERIMENTAL RESULTS \& ANALYSIS}

\section{A. Single Microphone Far-field Noise Spectrum}

Before azimuthally decomposing the mid-acoustic field, it is instructive to first look at the far-field behaviour of the jet. Narrow-band far-field noise spectra are shown in Fig. 2 at $\theta=60^{\circ}$ and $90^{\circ}$ for a range of NPR. Typical BBSAN directivity and amplitude traits are clearly observed; the peak BBSAN frequency decreases as NPR and $\theta$ increase. Moreover, it can be seen that the intensity of BBSAN, represented by overall sound pressure level (OASPL), follows the well-known $\beta^{4}$ proportionality (Harper-Bourne and Fisher, 1973) for $\beta^{4}>0.5$. Strong screech peaks are also evident in the acoustic spectra. The presence of screech is known to affect BBSAN, lowering the BBSAN peak frequency for the same operating condition (André et al., 2013) and also modulating the lengthscales of velocity fluctuations in the jet plume (Tan et al., 2017). Since the nozzles were not designed with screech suppression features, comparison with other BBSAN studies in literature where screech was suppressed (André et al., 2013; Norum and Seiner, 1982; Savarese et al., 2013; Viswanathan, 2002), was not undertaken.

In Fig. 3 we show sound pressure level (SPL) as a function of polar angle $\left(60^{\circ}<\theta<120^{\circ}\right)$ and frequency $(0.1<S t<1.5)$ at three different NPRs. The BBSAN peak frequency predictions from Eq. 1 are also plotted for comparison. Both $N P R=3.10$ and 3.67, in Fig. 3(b) and 3(c) respectively, display agreement with Eq. 1, where we have defined the convection velocity as $u_{c}=0.7 u_{j}$ and the shock-cell spacing $L_{1}$ using the vortex sheet approximation from the Pack and Prandtl (P\&P) model (Pack, 1950). The presence of a higherorder shock-cell mode $(n=2)$ is evident as the second BBSAN lobe. There is a slight underprediction of BBSAN frequency for $N P R=2.25$ (Fig. 3(a)), possibly due to the mismatch in shock-cell spacing between the P\&P model and experiment. Discrete screech peaks are also observed. Mixing noise is not prominent as this noise component has only small contribution at sideline and upstream positions (Sinha et al., 2014). The results from far-field measurements are in agreement with wellvalidated models from literature and give us confidence in the mid-field measurements presented below.

\section{B. Azimuthal Modal Analysis of the Mid-field}

Following the method shown in Appendix C, we obtain the azimuthal decomposition of the radiated noise. Due to the microphone array being only $11 D$ from the jet axis, the origin of the polar angle $\theta$ is now taken at a downstream location of $x_{0}=5 D$, in similar fashion to Gojon and Bogey (2017). The origin is redefined in an effort to more faithfully capture the origin of the acoustic source, and is an indirect demonstration that BB$\mathrm{SAN}$ is generated at the downstream shock-cells (Tam et al., 1985). Sound pressure level measurements are only shown up to $S t=1.5$ due to the upper frequency limit of the microphones. Hereafter, we shall focus only on the BBSAN component in our discussion.

\section{Azimuthally Decomposed Spectra}

The acoustic pressure spectra at $\theta=80^{\circ}, 95^{\circ}$ and $115^{\circ}$, for the range of NPRs in Table I, is shown in Fig. 4. The first four azimuthal modes $(m=0, \pm 1, \pm 2, \pm 3)$ are shown along with the full signal. For $m>0$, we combine both the negative and positive components (Eq. C8) and hence drop the ' \pm ' for compactness. The BBSAN lobe is present for all azimuthal modes and follows the known trends as Mach number and observer location vary. For both $N P R=3.10$ and 3.67 , the lobe due to the second shock-cell mode, viewed at the sideline and upstream angles, is clearly present.

We first focus on the relative importance of each azimuthal mode across the frequency range of interest. At low frequencies $(S t \lesssim 0.6-0.7)$, the single helical mode is dominant. There is a clear distinction between each successive mode $(m>1)$, with decreasing amplitude as mode number increases. The separation between modes becomes less pronounced as frequency increases. Fig. 4 also clearly shows that $m=0$ is not the dominant azimuthal mode for the angles of interest. The sound field of the axisymmetric mode is on average $3-4 \mathrm{~dB} / \mathrm{St}$ less than the dominant mode(s). This observation is also consistent with subsonic jet noise and source models; higher azimuthal modes ( $m=1$ and 2 ) become increasingly important at higher frequencies and for positions further upstream, while downstream noise is dominated by mode $m=0$ (Jordan and Colonius, 2013).

At $S t \approx 0.6-0.7$, the distinction between non-zero azimuthal modes is absent and we observe an approximately equal contribution from modes $m=1,2$ and 3 . This trend is observed at all three NPRs shown and continues as mode number increases (not shown for brevity). The increasing contribution from successive modes as frequency increases is consistent with the findings of Kuo et al. (2013) and Vold et al. (2012), who measured the same behaviour in both ideally-expanded and overexpanded jets.

By comparing these results against well-known BBSAN trends, we arrive at some observations of interest. Depending on the frequencies of the BBSAN lobe, the relative contribution of each azimuthal mode varies. For instance, looking at the $N P R=2.25$ jet at $\theta=80^{\circ}$, the $m=1,2$ and 3 azimuthal modes are of similar strength for the BBSAN lobe, while those same modes are distinct (with $m=1$ being the strongest) at the lobe for the $N P R=3.67$ case at $\theta=115^{\circ}$. A similar trend is also observed when comparing the azimuthal content of higher frequency secondary lobes $(n=2)$ to the primary one $(n=1)$.

The trends observed in Fig. 4 are shown in a more comprehensive manner in the $S t-\theta$ contour diagrams in Fig. 5. Decomposed acoustic spectra up to $m=4$ are shown between $50^{\circ}<\theta<125^{\circ}$. For both $N P R=3.10$ and 3.67 jets, the BBSAN directivity of the primary $(n=1)$ and secondary $(n=2)$ peaks agrees favourably 


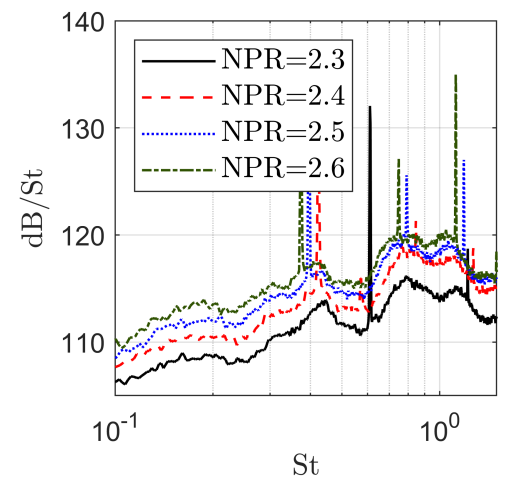

(a)

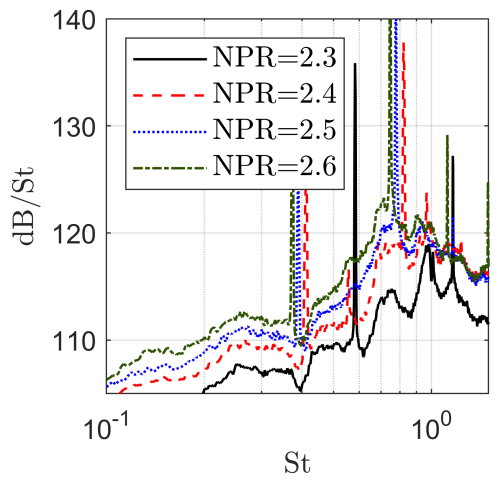

(b)

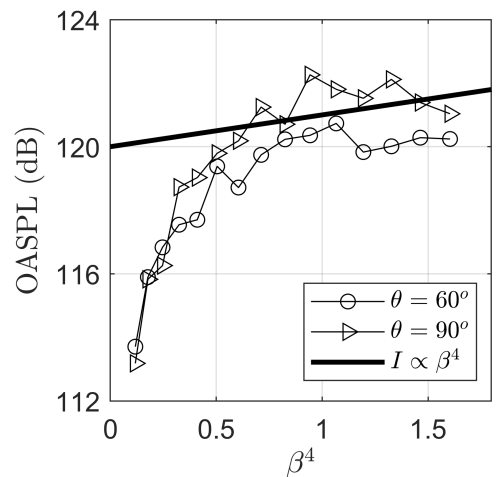

(c)

FIG. 2. (Colour Online) Effect of changing NPR at (a) $\theta=60^{\circ}$, (b) $\theta=90^{\circ}$ and the dependence on $\beta^{4}$ as a function of $M_{j}$.

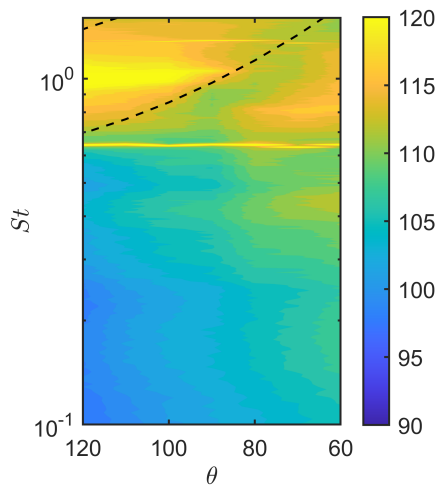

(a)

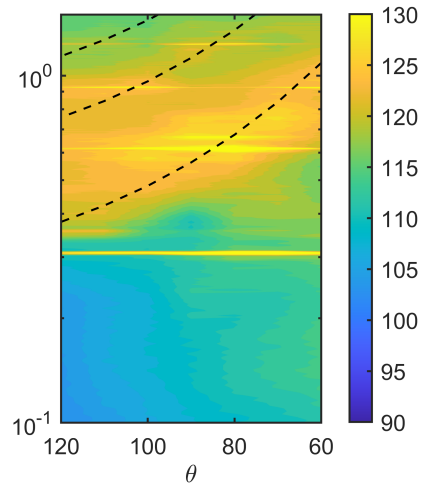

(b)

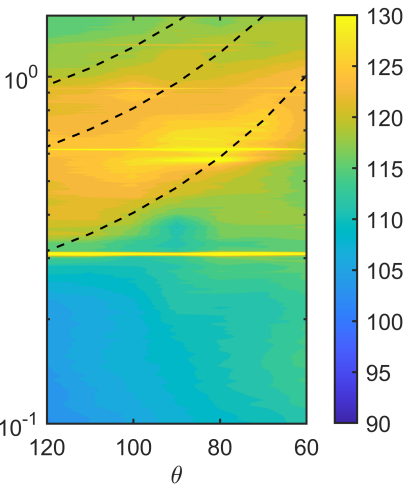

(c)
FIG. 3. (Colour Online) Directivity contour plots for power spectral density (PSD) in the far-field $(R=50 D)$ for NPRs (a) 2.25 , (b) 3.10 and (c) 3.67 . Polar angles taken with respect to downstream jet axis and contours are in $\mathrm{dB} /$ St. Dashed lines indicate peak frequency predictions from Eq. 1. with Eq. 1 (dashed lines). For $N P R=2.25$, the experimental peak frequency is higher than that suggested by Eq. 1, as expected from the far-field data in Fig. 3(a). The same inputs as section III A were used in Eq. 1 for all azimuthal modes. This suggests that the instability waves associated with each azimuthal mode convect at a similar velocity.

From Eq. 1, the BBSAN lobe shifts upward as Mach number decreases and observer position moves downstream. Hence, as $\theta$ and NPR decrease, the azimuthal content of BBSAN becomes increasingly multi-modal. Fig. 5 illustrates this trend. At low Mach numbers $(N P R=2.25)$, since the BBSAN lobe is above $S t \approx 0.7$, there is largely equal contribution from the first four nonzero modes. At higher Mach numbers, the BBSAN lobe shifts down and we see a difference in modal behaviour. At $N P R=3.67$, BBSAN that peaks at sideline and upstream angles $\left(\theta>90^{\circ}\right)$ is dominated by $m=0,1$ and 2 . However, at lower emission angles $\left(\theta<90^{\circ}\right)$, the BBSAN lobe shifts to higher frequencies and the contribution from modes $m=3$ and 4 is non-negligible. Similar behaviour is also seen for $N P R=3.1$. This trend is in agreement with Arroyo and Moreau (2019) where LES of a non-screeching underexpanded jet with an ideallyexpanded Mach number of $M_{j}=1.15$ was investigated. Although the experimental measurements of this study are conducted in the presence of screech, this does not seem to affect the frequency dependence of the azimuthal modes that contribute to BBSAN.

In regions where the difference between azimuthal modes is pronounced (low frequency, upstream angles), the acoustic field exhibits low-rank behaviour. Here, 'low-rank' is referred to as a rank-N truncation of the azimuthal Fourier basis which provides an approximate description of the sound field to within $1 \mathrm{~dB} /$ St precision of the full-rank data. The connection between BBSAN directivity and variation in azimuthal modal quality is further explored in the following section.

\section{Acoustic Field Reconstruction}

To investigate the relevance of subsequent lowerorder azimuthal modes to the full BBSAN signal as a function of $\theta$ and NPR, we perform reconstructions of the 

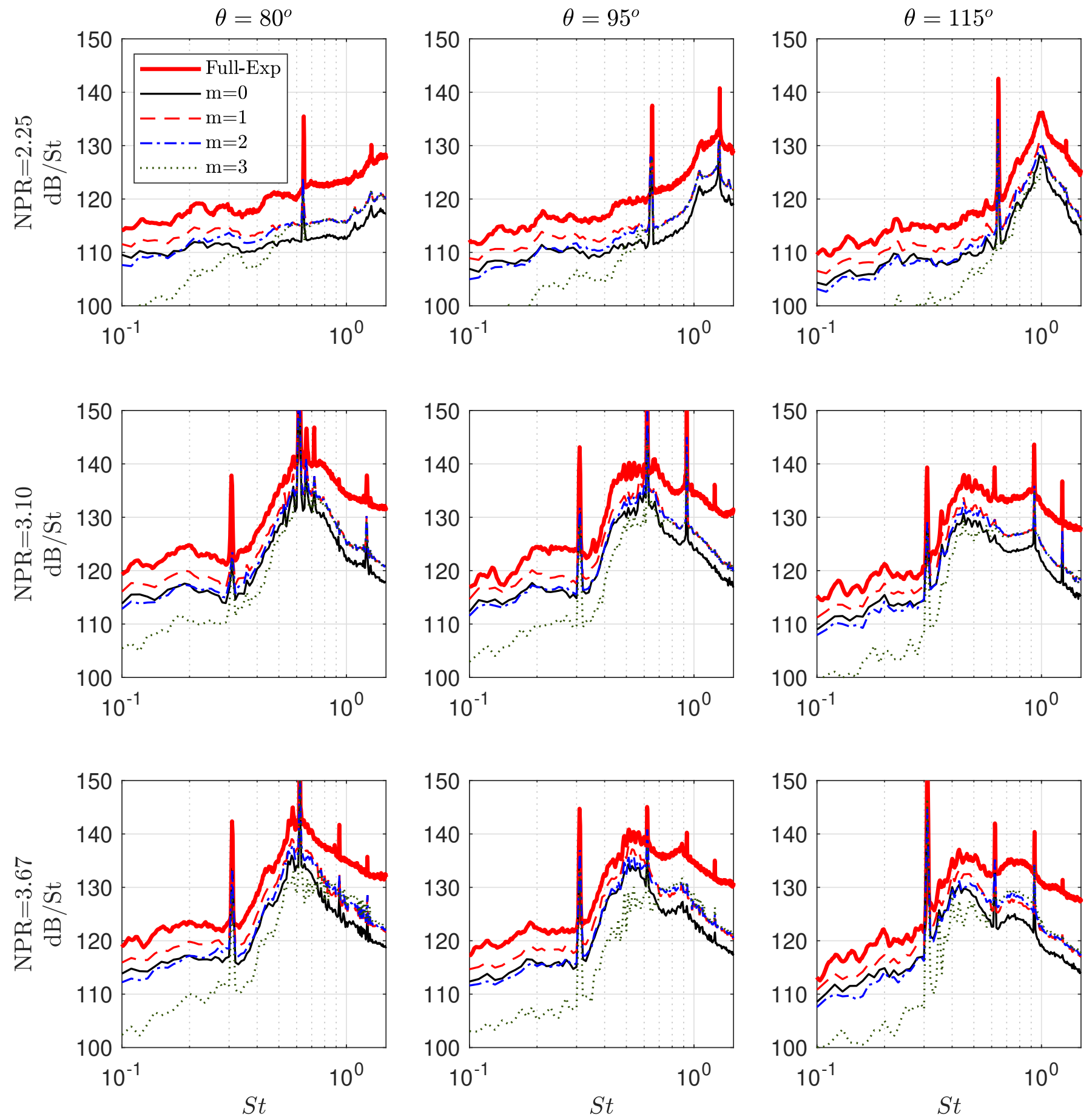

FIG. 4. (Colour Online) Decomposed acoustic spectra in the mid-field $(R=11 D)$ for different NPRs; $N P R=2.25$ (upper row), 3.10 (middle row) and 3.67 (lower row). Polar angles taken with respect to downstream jet axis; $\theta=80^{\circ}$ (left column), $\theta=95^{\circ}$ (middle column) and $\theta=115^{\circ}$ (right column).

acoustic field using an increasing number of azimuthal modes (Eq. C6). The upper row of Fig. 6 contains rank maps showing the number of modes required to attain $1 \mathrm{~dB} / \mathrm{St}$ precision for the total signal. The modes are added successively. The solid red contour provides a visual aid for the position of the BBSAN lobe. As expected from Fig. 4 , a single-mode $(m=0)$ representa- tion of the acoustic field is insufficient. At low frequencies $(S t \lesssim 0.6-0.7)$, apart from some inconsistencies for the $N P R=3.10$ case, low-rank behaviour is observed, with the combination of 2-3 modes sufficient to recover the full signal. Comparing this trend with the position of the BBSAN lobes, we hence observe low-rank behaviour for $N P R=3.10$ and $3.67 ; 2-3$ modes again provide a 


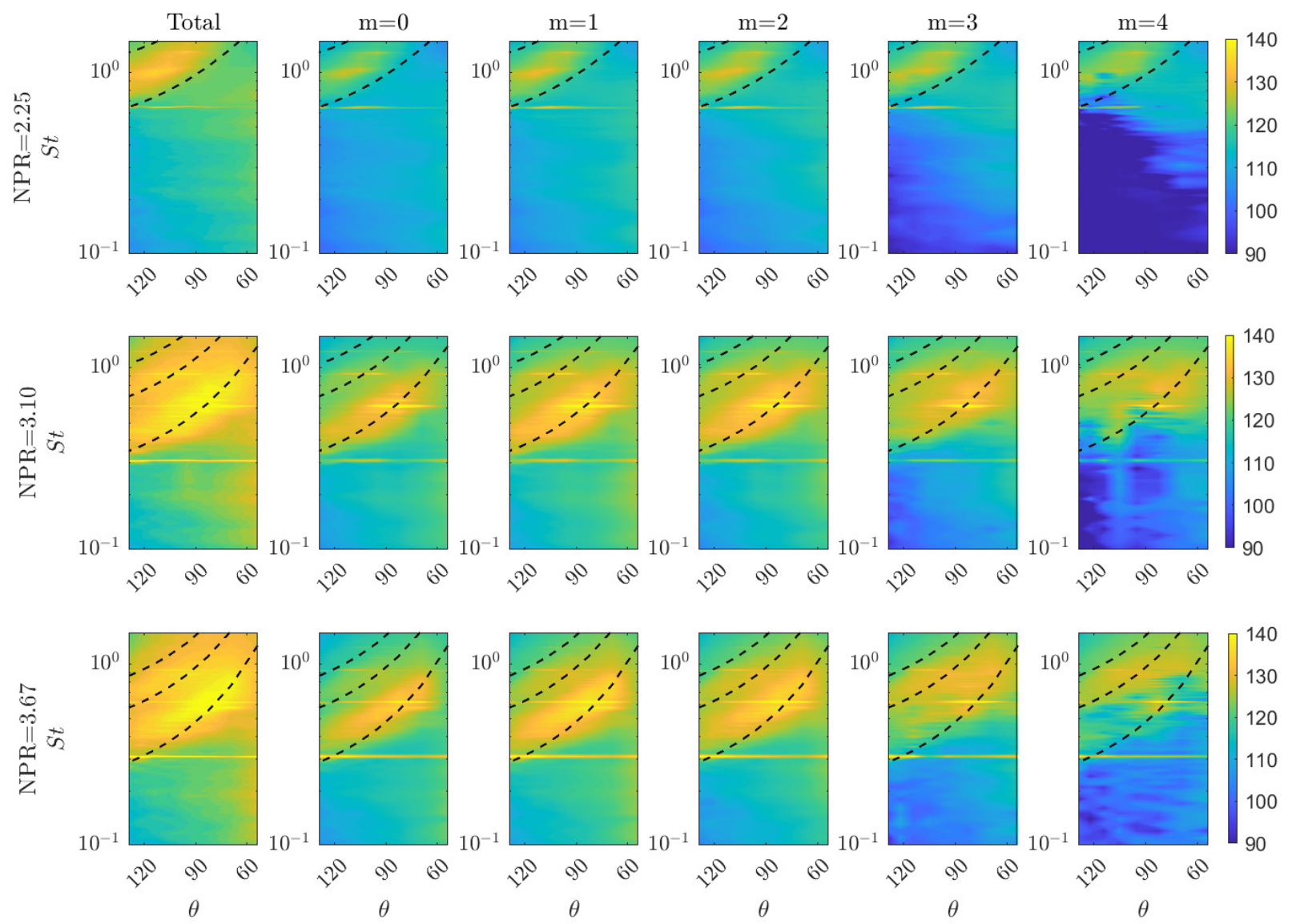

FIG. 5. (Colour Online) Full and decomposed spectra in the mid-field $(R=11 D)$ for different conditions; $N P R=2.25$ (upper row), $N P R=3.10$ (middle row) and $N P R=3.67$ (lower row). Polar angles taken with respect to downstream jet axis and contours are in $\mathrm{dB} /$ St. Dashed lines indicate peak frequency predictions from Eq. 1.

sufficient approximation for BBSAN. As alluded to in section IIIB 1, however, at lower emission angles the number of required modes increases. For example, at $N P R=3.10$, five modes are required at $\theta=70^{\circ}$, while only three modes are used at $\theta=100^{\circ}$ to obtain the same precision. The latter observation, however, does not apply at $N P R=2.25$, where the higher-frequency lobe consistently requires 4-5 modes to capture the total BBSAN amplitude.

To further illustrate this behaviour, reconstructed acoustic spectra at $\theta=95^{\circ}$ are shown in the lower row of Fig. 6. The corresponding position on the rank maps is indicated by the vertical dashed line. While convergence is rapid at low frequencies, the low-rank approximation deteriorates as frequency increases. Consequently, we observe that the secondary lobe is of increased modal complexity relative to the primary lobe (Fig. $6 \mathrm{~b}$ and c). To summarise, BBSAN can be approximated by an azimuthally low-rank approximation. As the peak frequency varies, the exact number of modes required for reconstruction of the total sound field will depend on both the operating condition of the jet and the observer position, with an increasing number of modes required at higher frequency.

These observations suggest that previous instability wave BBSAN models neglected some relevant features. For example, the removal of azimuthal dependence by Tam (1987) does not adequately address the multi-modal behaviour at high frequencies. Similarly, we might infer that the truncation of modes to $m \leq 2$ by Ray and Lele (2007) likely contributed to the observed 'missing sound' at high frequencies. These approximations may explain why such models either had poor amplitude predictions at high frequencies, or resorted to empiricism to match the total measured sound field. A more insightful means of validating such models may be to undertake azimuthal mode-by-mode comparisons, similar to the process of Cavalieri et al. (2012) for subsonic jets.

\section{SUMMARY \& OUTLOOK}

Acoustic measurements were obtained from an underexpanded jet operating at three conditions. Far-field single microphone measurements were acquired to val- 

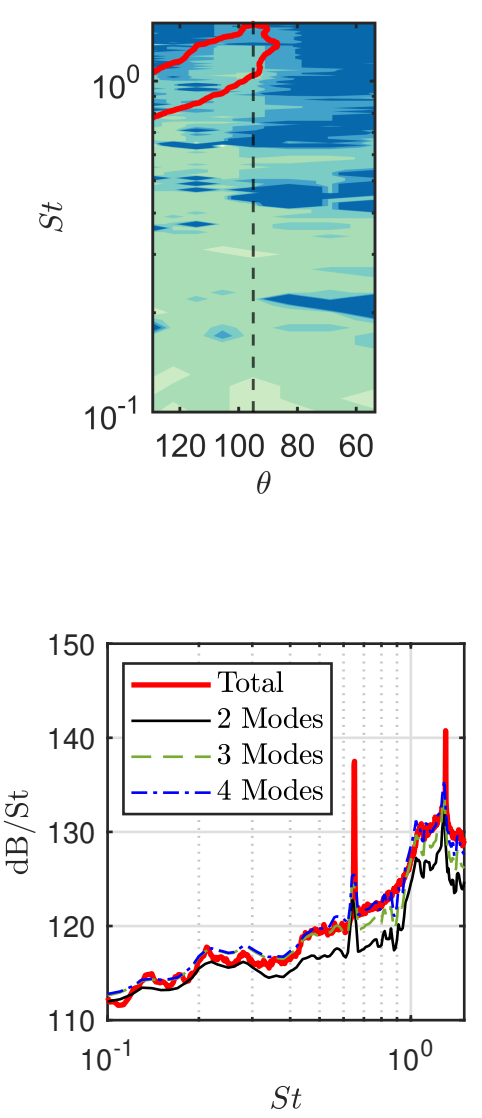

(a) $N P R=2.25$
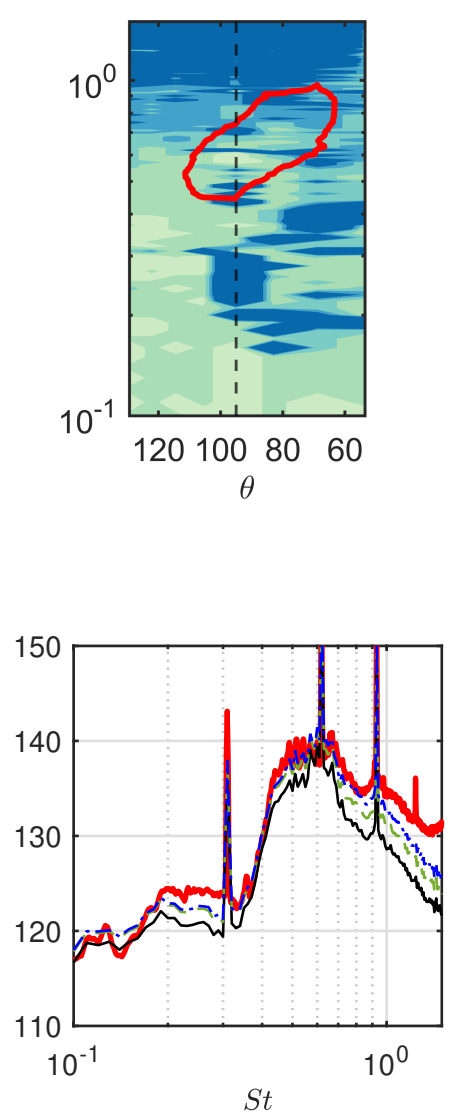

(b) $N P R=3.10$
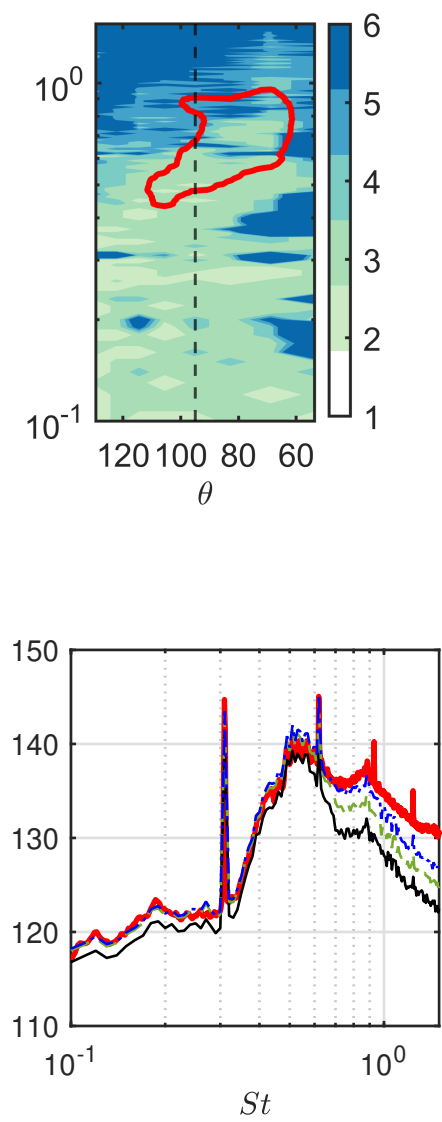

(c) $N P R=3.67$

FIG. 6. (Colour Online) Reconstruction of the acoustic field with modes added successively $(m=0, \pm 1, \pm 2 \ldots)$. Top row; $S t-\theta$ maps displaying the number of modes required to reconstruct the total acoustic signal to within $1 \mathrm{~dB} / \mathrm{St}$ for $N P R=$ a) 2.25 , b) 3.10 and c) 3.67 . The $130 \mathrm{~dB} /$ St contour (red line) identifies the primary BBSAN lobe seen in Fig. 5 . The vertical dashed lines locate $\theta=95^{\circ}$. Bottom row; Reconstruction of acoustic spectrum at $\theta=95^{\circ}$ with increasing number of modes.

idate measurements from the new facility. Using two microphones mounted on a traversable array, the sound field was decomposed for the purposes of exploring the modal composition of BBSAN.

The single microphone measurements were in agreement with previous observations. Strong screech peaks were observed and the well-known BBSAN directivity shape was recovered, in agreement with Tam and Tanna (1982). The BBSAN amplitude was also found to follow the $\beta^{4}$ scaling.

Using a similar methodology to Sinha et al. (2018), azimuthally-decomposed acoustic spectra were obtained. It was found that, unlike in subsonic and ideallyexpanded jets where the dominant sound radiation is in the downstream direction, the axisymmetric mode is not the dominant contribution to BBSAN in the acoustic field. Rather, the $m=1$ mode is the most important for $S t \lesssim 0.7$. Further, we show that modes $m>1$ are increasingly important for higher frequencies. Successive reconstructions of the acoustic field demonstrate that approximately 3-5 azimuthal modes are required to recover the total BBSAN signal; the minimum number of requisite modes varies depending on the peak frequency. When correlated with the known directivity of BBSAN, we arrive at an interesting corollary; the total BBSAN sound field becomes progressively low-rank in azimuth as polar angle and Mach number increase. For the secondary lobe, at higher frequencies than the primary one, we clearly see higher-rank behaviour.

From a modelling perspective, the findings are consistent with the predictions made by wavepacket BBSAN models. In such models, the shock-cells are axisymmetric and assumed to be stationary, while the frequency and azimuthal characteristics are solely defined by the wavepacket. It is well-known that the source location shifts towards the nozzle as frequency increases (Suzuki, 2016). Previous parabolised stability equation (PSE) calculations of hydrodynamic wavepackets in supersonic jets (Ray and Lele, 2007; Rodríguez et al., 2015) have shown that the spatial extent of wavepackets contracts as azimuthal mode number increases, shifting the peak of the envelope closer to the nozzle exit. Hence, only for the 
high frequency BBSAN components can wavepackets of higher-order modes be acoustically efficient. Ray and Lele (2007) also found that the energy is more evenly distributed across azimuthal modes of instability waves as frequency increases, in accordance with our findings here.

The nontrivial contribution of higher-order azimuthal modes to the full spectrum necessitates that wavepacket models for BBSAN should be able to accurately predict their evolution, particularly for higher frequencies, as confidently as for the lower-order modes. Sasaki et al. (2017) have demonstrated the validity of wavepacket models up to a Strouhal number of $S t=4.0$ and azimuthal wavenumber $m=4$ for a $M=0.9$ jet. This suggests wavepackets are well-placed to model the large-scale structures over the BBSAN spectral range. While outside the scope of the present study, there is clear motivation for future work on the construction and refinement of these wavepacket models for shockcontaining jets.

The results presented not only directly address the issue of the lack of azimuthally-decomposed acoustic data, but also shed light on the relative contribution of each azimuthal mode to the full BBSAN signature. The importance of higher-order modes, including their relevance in shaping overall BBSAN directivity, should be addressed in future models.

\section{ACKNOWLEDGMENTS}

This research is supported by an Australian Government Research Training Program (RTP) Scholarship and the Endeavour Research Leadership Award - an Australian Government initiative. The authors also received funding from the Australian Research Council through the Discovery Projects scheme. M.H.W would also like to acknowledge Mr Danny Lynch, Mr Phil Nicholson and the support of the Monash Mechanical and Aerospace Technical Services Group in the construction of SJAF.

\section{APPENDIX A:}

The method of Ahuja (2003) is used to calibrate the anechoic chamber and determine its acoustic 'cleanliness'. For a point source, the measured sound amplitude should obey the inverse-square law as distance increases in any direction. Apart from the removal of the nozzle and azimuthal ring, no further modifications were made inside the chamber. A $45 \mathrm{~mm}$ diameter speaker, with a range of $140-20,000 \mathrm{~Hz}$, is placed along the jet centreline at the same position as the nozzle exit. The same G.R.A.S. Type 46BE 1/4" microphones were used and mounted on a traverse line at different directions from the source. To determine the deviation from the inversesquare law, the method prescribed by Cunefare et al. (2003) was used. The theoretical free-field SPL $\left(S P L_{p}\right)$ at distance $r_{i}$ is given by

$$
S P L_{p}\left(r_{i}\right)=20 \log _{10}\left[\frac{a}{r_{i}-r_{0}}\right],
$$
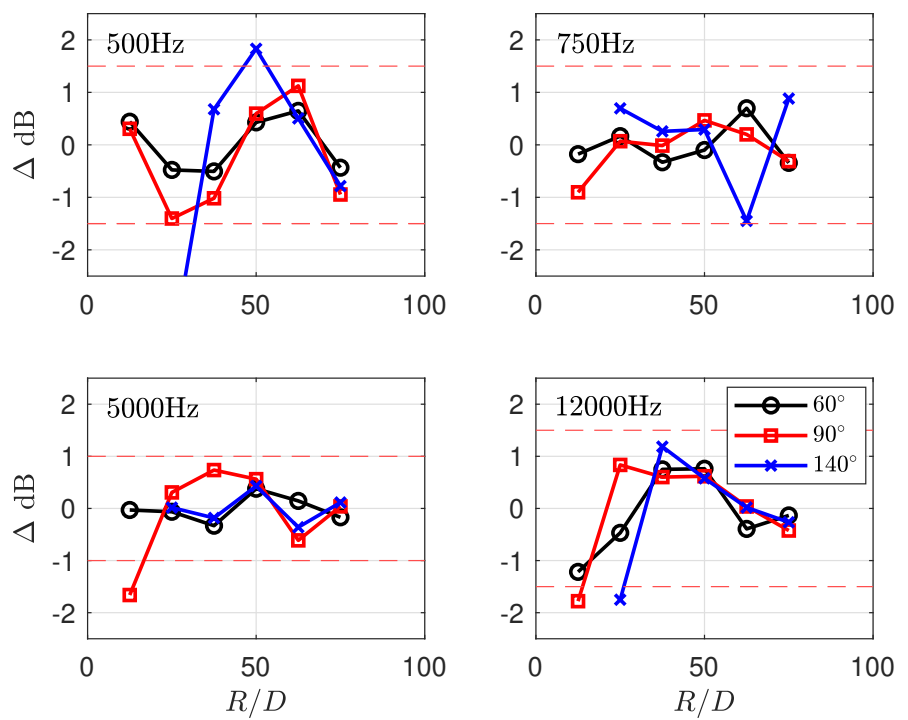

FIG. 7. Deviation from the inverse-square law at three different angles for a range of frequencies and polar angles. Red dashed line indicates maximum permissible deviation as specified by ISO 3745 .

where $a$ and $r_{0}$ are the apparent acoustic source strength and the offset distance between the physical location of the source and its acoustic centre respectively. For $N$ number of measurements in the radial direction, the values of $a$ and $r_{0}$ are given as

$$
\begin{array}{r}
a=\frac{\left(\sum_{i=1}^{N} r_{i}\right)^{2}-N \sum_{i=1}^{N} r_{i}^{2}}{\sum_{i=1}^{N} r_{i} \sum_{i=1}^{N} q_{i}-N \sum_{i=1}^{N} r_{i} q_{i}} \\
r_{0}=\frac{\sum_{i=1}^{N} r_{i} \sum_{i=1}^{N} r_{i} q_{i}-\sum_{i=1}^{N} r_{i}^{2} \sum_{i=1}^{N} q_{i}}{\sum_{i=1}^{N} r_{i} \sum_{i=1}^{N} q_{i}-N \sum_{i=1}^{N} r_{i} q_{i}}
\end{array}
$$

where $q_{i}=10^{-0.05\left(S P L_{p i}\right)}$. The deviation in $\mathrm{dB}$ between the measured $S P L_{p i}$ and theoretical value at distance $r_{i}$ is hence given by

$$
\triangle S P L_{p}\left(r_{i}\right)=S P L_{p i}-S P L_{p}\left(r_{i}\right) .
$$

The curves in Fig. 7 show that the frequencies of interest are within the required tolerance as specified in ISO 3745:2012 and confirms that the inverse-square law holds for far-field measurements. Some slight deviations are seen closer to the nozzle at upstream positions $(\theta=$ $140^{\circ}$ ) due to the presence of upstream piping.

\section{APPENDIX B:}

The following outlines the steps required to process microphone data to obtain the ensemble-averaged power spectral density (PSD) spectra shown in section III. All acoustic data were acquired at a sample rate of $200 \mathrm{kHz}$ for two seconds, giving a total of $N_{t}=4 \times 10^{5}$ sample points $P(t)$. The signal is checked for spurious trends and 
noise spikes, and a low-pass filter is applied at $80 \mathrm{kHz}$. The mean of the signal is removed and then de-trended using a linear regression line $\widetilde{P}(t)$

$$
p(t)=\left(P(t)-\frac{1}{N_{t}} \sum_{n=1}^{N_{t}} P(t)\right)-\widetilde{P}(t) .
$$

Before carrying out the Fast Fourier transforms (FFTs) of $p(t)$, to improve convergence, we separate the time signal into $N_{B}$ blocks of data each containing $N_{f f t}=$ 2048 points. To suppress side-lobe leakage, a Hanning window $H_{0}(t)$ is used. The FFT of each block $p_{k}(t)$ is then given by

$$
p_{k}(f)=\frac{\sqrt{8 / 3}}{N_{f f t}}\left(\operatorname{FFT}\left(H_{0}(t) p_{k}(t)\right)\right) .
$$

The power spectral density is computed by

$$
P S D(f)=\frac{1}{N_{B}} \sum_{k=1}^{K} p_{k}(f) p_{k}^{*}(f),
$$

where the signal has been averaged over $N_{B}=974$ blocks and ${ }^{*}$ denotes the complex conjugate. We convert the PSD into SPL using the classic definition and a reference pressure of $P_{\text {ref }}=20 \mu \mathrm{Pa}$ giving

$$
S P L(f)=10 \log _{10}\left(\frac{P S D(f)}{P_{\text {ref }}^{2}}\right) .
$$

The presented SPL is in units of $\mathrm{dB} / \mathrm{St}$ since the PSD is nondimensionalised by the factor $U_{j} / D_{j}$, and frequency is thus defined as $S t=f D_{j} / U_{j}$.

\section{APPENDIX C:}

We present the methodology used to compute the two-point cross correlation matrix $R_{p p}\left(\phi_{1}, \phi_{2}\right)$. The Fourier-transformed signals from the two microphones (Eq. B2), are used to compute the cross spectrum,

$$
R_{p p}\left(\phi_{1}, \phi_{2} ; f\right)=\frac{1}{N_{B}} \sum_{k=1}^{K} p_{k}\left(\phi_{1} ; f\right) p_{k}^{*}\left(\phi_{2} ; f\right),
$$

where $\psi=\phi_{2}-\phi_{1}$ and we drop the $x$ and $r$ coordinates for compactness as both microphones are on the same circumferential plane. The azimuthal homogeneity of the round jet allows $R_{p p}$ to only depend on $\psi$.

The increment in $\phi$ is fixed at $10^{\circ}$, but a finer discretisation is desired for our analysis. We employ interpolation to reconstruct $R_{p p}$ for each desired $S t$. We note that $R_{p p}$, from Eq. C1, is a complex quantity and can be separated into the synchronous (real) and asynchronous (imaginary) component,

$$
R_{p p}(\psi ; f)=a(\psi ; f)+i b(\psi ; f) .
$$

The real component, $a(\psi ; f)$ is a symmetric function and hence a sinc function was found to be an appropriate fit
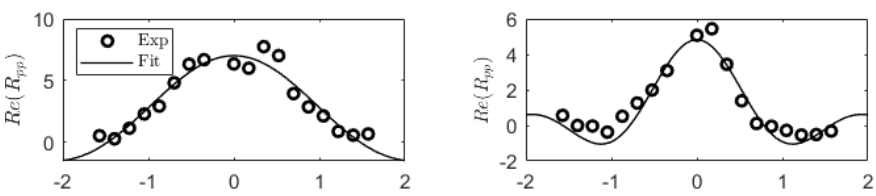

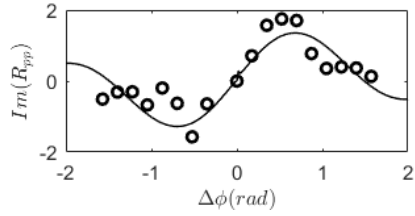

(a)

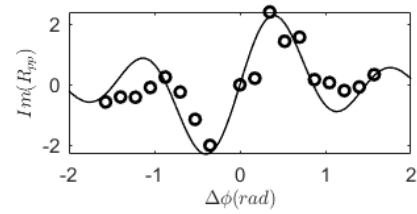

(b)
FIG. 8. Example of an interpolated fit to the $R_{p p}$ two-point data as a function of azimuthal separation for $N P R=3.67$ at $\theta=95^{\circ}$ for a) $S t=0.2$ and b) $S t=0.7$.

for a wide range of frequencies. For the imaginary component, $b(\psi ; f)$ is antisymmetric along the diagonal. The derivative of the sinc function was used to fit the imaginary component well. The interpolated fits are summarised as

$$
\begin{aligned}
& a(\psi ; f) \approx A \operatorname{sinc}(B \psi) \\
& b(\psi ; f) \approx \frac{(C(D \psi \cos (D \psi)-\sin (D \psi)))}{D \psi^{2}},
\end{aligned}
$$

where $A, B, C, D$ are fitting parameters. An example of the raw data $R_{p p}$ with an interpolated fit $\widetilde{R}_{p p}$ is shown in figure 8 . Where a sinc function was found to be inappropriate, the values at the grid points are connected with a linear fit. We note here that the goal is to have a curve that is faithful to the measured data rather than to infer physical meaning from the fit itself.

Using the interpolated fits $\widetilde{R}_{p p}$, the $m^{\text {th }}$ azimuthal Fourier mode is obtained by performing a Fourier transform in azimuth, giving

$$
\widetilde{R}_{p p}(f, m)=\frac{1}{2 \pi} \int_{-\pi}^{\pi} \widetilde{R}_{p p}\left(\phi_{1}, \phi_{2} ; f\right) e^{-i m \psi} d \psi,
$$

and its reconstruction is given by

$$
\widetilde{R}_{p p}(\psi ; f)=\sum_{m=-\infty}^{\infty} \widetilde{R}_{p p}(f, m) .
$$

where for the axisymmetric mode $(m=0)$,

$$
\widetilde{R}_{p p, 0}(\psi ; f)=\widetilde{R}_{p p}(f, m=0),
$$

and for $m \geq 1$

$$
\widetilde{R}_{p p, m}(\psi ; f)=\widetilde{R}_{p p}(f, m)+\widetilde{R}_{p p}(f,-m) .
$$

Using the circumferential isotropy and homogeneity properties (Michalke and Fuchs, 1975), the Fourier coefficients of $\widetilde{R}_{p p}$ are equivalent to the PSD of pressure fluctuations when $\psi=0$, which gives

$$
P S D(f)=\widetilde{R}_{p p}(\psi=0 ; f),
$$


and hence the amplitude $\left|\widetilde{R}_{p p, m}(\psi=0 ; f)\right|$ is substituted into Eq. B4 to compute the power spectrum for each azimuthal mode.

Ahuja, K. (2003). "Designing clean jet-noise facilities and making accurate jet-noise measurements," International Journal of Aeroacoustics 2(3), 371-412.

André, B., Castelain, T., and Bailly, C. (2013). "Broadband shockassociated noise in screeching and non-screeching underexpanded supersonic jets," AIAA journal .

Arroyo, C. P., and Moreau, S. (2019). "Azimuthal mode analysis of broadband shock-associated noise in an under-expanded axisymmetric jet," Journal of Sound and Vibration 449, 64-83.

Bogey, C., Marsden, O., and Bailly, C. (2012). "Effects of moderate reynolds numbers on subsonic round jets with highly disturbed nozzle-exit boundary layers," Physics of Fluids 24(10), 105107.

Cavalieri, A. V., Jordan, P., Colonius, T., and Gervais, Y. (2012).

"Axisymmetric superdirectivity in subsonic jets," Journal of fluid Mechanics 704, 388-420.

Cavalieri, A. V., Jordan, P., and Lesshafft, L. (2019). "Wavepacket models for jet dynamics and sound radiation," Applied Mechanics Reviews 71(2), 020802.

Cunefare, K. A., Biesel, V. B., Tran, J., Rye, R., Graf, A., Holdhusen, M., and Albanese, A.-M. (2003). "Anechoic chamber qualification: Traverse method, inverse square law analysis method, and nature of test signal," The Journal of the Acoustical Society of America 113(2), 881-892.

Edgington-Mitchell, D. (2019). "Aeroacoustic resonance and selfexcitation in screeching and impinging supersonic jets-a review," International Journal of Aeroacoustics 18(2-3), 118-188.

Edgington-Mitchell, D., Oberleithner, K., Honnery, D. R., and Soria, J. (2014). "Coherent structure and sound production in the helical mode of a screeching axisymmetric jet," Journal of Fluid Mechanics 748, 822-847.

Gojon, R., and Bogey, C. (2017). "Numerical study of the flow and the near acoustic fields of an underexpanded round free jet generating two screech tones," International Journal of Aeroacoustics 16(7-8), 603-625.

Harper-Bourne, M., and Fisher, M. (1973). "The noise from shock waves in supersonic jets," AGARD-CP-131 11, 1-13.

Huber, J., Fleury, V., Bulté, J., Laurendeau, E., and Sylla, A. A. (2014). "Understanding and reduction of cruise jet noise at aircraft level," International Journal of Aeroacoustics 13(1-2), 6184.

ISO 3745:2012 (2012). "ISO 3745: Acoustics — Determination of sound power levels and sound energy levels of noise sources using sound pressure - Precision methods for anechoic rooms and hemi-anechoic rooms," Standard.

Jordan, P., and Colonius, T. (2013). "Wave packets and turbulent jet noise," Annual Review of Fluid Mechanics 45, 173-195.

Knast, T., and Edgington-Mitchell, D. (2017). "Design of a supersonic wind tunnel facility," in 17th Australian International Aerospace Congress: AIAC 2017, Engineers Australia, Royal Aeronautical Society, p. 516

Kuo, C.-W., Buisson, Q., McLaughlin, D. K., and Morris, P. J. (2013). "Experimental investigation of near-field pressure fluctuations generated by supersonic jets," in 19th AIAA/CEAS aeroacoustics conference, p. 2033.

Lele, S. (2005). "Phased array models of shock-cell noise sources," in 11th AIAA/CEAS aeroacoustics conference, p. 2841.

Lighthill, M. J. (1952). "On sound generated aerodynamically. i. general theory," in Proceedings of the Royal Society of London A: Mathematical, Physical and Engineering Sciences, The Royal Society, Vol. 211, pp. 564-587.

Michalke, A. (1970). "A wave model for sound generation in circular jets," Technical Report (Deutsche Forschungs-und Versuchsanstalt für Luft-und Raumfahrt, eV).

Michalke, A., and Fuchs, H. (1975). "On turbulence and noise of an axisymmetric shear flow," Journal of Fluid Mechanics 70(1), 179-205.

Morel, T. (1975). "Comprehensive design of axisymmetric wind tunnel contractions," Journal of Fluids Engineering 97(2), 225-
233

Norum, T., and Seiner, J. (1982). "Measurements of mean static pressure and far field acoustics of shock containing supersonic jets," Technical Report (TM 84521, NASA).

Ozawa, Y., Nonomura, T., Oyama, A., and Asai, K. (2020). "Effect of the reynolds number on the aeroacoustic fields of a transitional supersonic jet," Physics of Fluids 32(4), 046108.

Pack, D. (1950). "A note on prandtl's formula for the wave-length of a supersonic gas jet," The Quarterly Journal of Mechanics and Applied Mathematics 3(2), 173-181.

Raman, G. (1999). "Supersonic jet screech: half-century from powell to the present," Journal of Sound and Vibration 225(3), 543571

Ray, P., and Lele, S. K. (2007). "Sound generated by instability wave/shock-cell interaction in supersonic jets," Journal of fluid mechanics 587, 173-215.

Rodríguez, D., Cavalieri, A. V., Colonius, T., and Jordan, P. (2015). "A study of linear wavepacket models for subsonic turbulent jets using local eigenmode decomposition of piv data," European Journal of Mechanics-B/Fluids 49, 308-321.

Sasaki, K., Cavalieri, A. V., Jordan, P., Schmidt, O. T., Colonius, T., and Brès, G. A. (2017). "High-frequency wavepackets in turbulent jets," Journal of Fluid Mechanics 830, R2.

Savarese, A., Jordan, P., Girard, S., Collin, E., Porta, M., and Gervais, Y. (2013). "Experimental study of shock-cell noise in underexpanded supersonic jets," AIAA Paper 2080, 2013.

Seiner, J. M., and Yu, J. C. (1984). "Acoustic near-field properties associated with broadband shock noise," AIAA journal 22(9), $1207-1215$.

Sinha, A., Rodríguez, D., Brès, G. A., and Colonius, T. (2014). "Wavepacket models for supersonic jet noise," Journal of Fluid Mechanics 742, 71-95.

Sinha, A., Towne, A., Colonius, T., Schlinker, R. H., Reba, R., Simonich, J. C., and Shannon, D. W. (2018). "Active control of noise from hot supersonic jets," AIAA Journal 56(3), 933-948.

Suzuki, T. (2016). "Wave-packet representation of shock-cell noise for a single round jet," AIAA Journal 54(12), 3903-3917.

Suzuki, T., and Colonius, T. (2006). "Instability waves in a subsonic round jet detected using a near-field phased microphone array," Journal of Fluid Mechanics 565, 197-226.

Tam, C. (1987). "Stochastic model theory of broadband shock associated noise from supersonic jets," Journal of Sound and Vibration 116(2), 265-302.

Tam, C. (1995). "Supersonic jet noise," Annual Review of Fluid Mechanics 27(1), 17-43.

Tam, C., Jackson, J., and Seiner, J. (1985). "A multiple-scales model of the shock-cell structure of imperfectly expanded supersonic jets," Journal of Fluid Mechanics 153, 123-149.

Tam, C., and Tanna, H. (1982). "Shock associated noise of supersonic jets from convergent-divergent nozzles," Journal of Sound and Vibration 81(3), 337-358.

Tan, D., Soria, J., Honnery, D., and Edgington-Mitchell, D. (2017). "Novel method for investigating broadband velocity fluctuations in axisymmetric screeching jets," AIAA Journal

Vaughn, A. B., Neilsen, T. B., Gee, K. L., Wall, A. T., Micah Downing, J., and James, M. M. (2018). "Broadband shock-associated noise from a high-performance military aircraft," The Journal of the Acoustical Society of America 144(3), EL242-EL247.

Viswanathan, K. (2002). "Quality of jet noise data: Issues, implications and needs," AIAA Paper 365.

Vold, H., Shah, P., Morris, P., Du, Y., and Papamoschou, D. (2012). "Axisymmetry and azimuthal modes in jet noise," in 18th AIAA/CEAS Aeroacoustics Conference (33rd AIAA Aeroacoustics Conference), p. 2214.

Wong, M. H., Edgington-Mitchell, D. M., Honnery, D., Cavalieri, A. V., and Jordan, P. (2019a). "A parabolised stability equation based broadband shock-associated noise model," in 25th AIAA/CEAS Aeroacoustics Conference, p. 2584.

Wong, M. H., Jordan, P., Honnery, D. R., and Edgington-Mitchell, D. (2019b). "Impact of coherence decay on wavepacket models for broadband shock-associated noise in supersonic jets," Journal of Fluid Mechanics 863, 969-993. 